\title{
Finding the sweet spot in preoperative assessment
}

\author{
Peter T. Choi, MD • Donald E. Griesdale, MD
}

Received: 30 January 2014/ Accepted: 17 February 2014/Published online: 1 March 2014

(C) Canadian Anesthesiologists' Society 2014

Diabetes mellitus ("diabetes") is a chronic condition with a substantive worldwide burden. Last year, the global prevalence of diabetes was 382 million people ( $8.3 \%$ of the world's population); and by 2035 , the prevalence is predicted to increase to 592 million. ${ }^{1}$ In Canada, nearly 2.4 million people, or $6.7 \%$ of the population, are diagnosed with diabetes. Importantly, $20 \%$ of diabetes remains undiagnosed. ${ }^{2}$ Within American, European, and Oceanic countries in 2010, Canada had the third highest prevalence amongst individuals aged 20-79 $\mathrm{yr}^{2}$

The economic cost of diabetes on an individual healthcare system and societal level are immense. Diabetes and its longterm complications result in increased rates in both hospitalization and premature death. ${ }^{2}$ The Public Health Agency of Canada estimated the national cost of diabetes to be $\$ 2.5$ billion in $2000 .^{2}$ This conservative estimate did not

P. T. Choi, MD $(\varangle)$ · D. E. Griesdale, MD

Department of Anesthesiology, Pharmacology and Therapeutics,

The University of British Columbia, 3300 - 910 West 10th

Avenue, Vancouver, BC V5X 4E3, Canada

e-mail: petert.choi@vch.ca

P. T. Choi, MD - D. E. Griesdale, MD

Department of Anesthesiology and Perioperative Care,

Vancouver General Hospital, Vancouver, BC, Canada

P. T. Choi, MD - D. E. Griesdale, MD

Center for Clinical Epidemiology and Evaluation, Vancouver

Coastal Health Research Institute, Vancouver, BC, Canada

P. T. Choi, MD

School of Population and Public Health, The University of British Columbia, Vancouver, BC, Canada

D. E. Griesdale, MD

Division of Critical Care Medicine, The University of British

Columbia, Vancouver, BC, Canada include costs from long-term complications or costs from other chronic diseases associated with diabetes.

In the perioperative setting, diabetes is associated with increased morbidity and mortality, ${ }^{3-5}$ especially in the presence of hyperglycemia. Preoperative identification of patients with undiagnosed diabetes and patients with poor glycemic control of known diabetes may guide decisions related to perioperative and long-term glycemic control. Identification of this latter group is especially important as prior studies show reduced risk of microvascular complications with improved glycemic control over a long-term basis. $^{6}$ Unfortunately, achievement of satisfactory glycemic control remains challenging. In one Canadian study, only $39 \%$ of participants maintained the targeted level of $7 \%$ or less for glycosylated hemoglobin A1c (HbA1c) over a one-year period. ${ }^{7}$ In one cohort study, only $22.4 \%$ of patients with poorly controlled diabetes admitted to hospital received some change in diabetic management by the time of discharge. ${ }^{8}$

In this issue, Koumpan et al. present a prospective observational study to determine the prevalence of chronic hyperglycemia in preoperative patients without a diagnosis of diabetes, the adequacy of glycemic control in preoperative patients with diabetes, and the diagnostic operating characteristics of random blood glucose and fasting blood glucose in the identification of patients with poor glycemic control. ${ }^{9}$ Participants were adults scheduled for assessment in the study site's preoperative clinic prior to elective surgery. An HbA1c of at least $6.5 \%$ was used to provisionally diagnose diabetes and serve as the gold standard for other measurements of glycemic control. The diagnosis of diabetes was based on the HbAlc values recommended by the Canadian Diabetes Association. ${ }^{10}$

The cohort consisted of 332 (82.6\%) participants without a diagnosis of diabetes and $70(17.4 \%)$ participants with known 
diabetes. Amongst the participants without diagnosed diabetes, the investigators found that " $23.2 \%$ (77/332) were at very high risk for developing diabetes (pre-diabetic), and $3.9 \%$ (13/332) had a provisional diagnosis of diabetes" based on HbA1c values. 9 Amongst the participants with known diabetes, 55.7\% (39/70) had suboptimal glycemic control as defined by an HbA1c of more than $7 \%$. The results confirm the prevalence of undiagnosed pre-diabetes and diabetes reported in earlier perioperative studies. ${ }^{11,12}$ The prevalence of suboptimal glycemic control amongst patients with diabetes is similar to previous epidemiological studies (49\% in Canada; $63.0 \%$ in United States $)^{13,14}$ and highlights the ongoing challenge of optimal long-term glycemic control.

The Table shows the diagnostic characteristics of random and fasting blood glucose in this study. When individuals with no prior diagnosis of diabetes were evaluated using HbA1c criteria, a new diagnosis of diabetes could be inferred in the presence of positive tests, i.e., if both random blood glucose and fasting blood glucose were specific for diabetes. Neither test was sensitive: a diagnosis of diabetes cannot be ruled out in the presence of a negative test. When the individuals with a history of diabetes were evaluated using HbA1c criteria, random blood glucose was specific but not sensitive for chronic suboptimal glycemic control, whereas fasting blood glucose was sensitive but not specific. The numbers are small in most of the diagnostic categories (true positive, false positive, false negative, and true negative) and result in wide confidence intervals for the estimates of the diagnostic characteristics; thus, caution should be taken when interpreting these results.

Several conclusions can be drawn if this study's findings are confirmed in larger cohorts. First, in patients without a history of diabetes, an elevated random or fasting blood glucose test is highly suspicious for diabetes and should lead to further diagnostic assessment and follow-up. Nevertheless, the absence of elevated results may not be reassuring, particularly in patients with risk factors for diabetes. ${ }^{9}$ Second, amongst patients with known diabetes, neither test alone is sufficient to determine the state of glycemic control. This observation is consistent with management of diabetes outside the perioperative setting when a single blood glucose test is insufficient to determine chronic glycemic control. Glycosylated hemoglobin remains a better test for diagnosing diabetes and determining the quality of long-term glycemic control.

As anesthesiologists, we may be tempted to downplay the implications of this study. Even with 402 participants, the sample size is small. Canadian guidelines recommend fasting blood glucose, which is fast and cheap compared with HbA1c. ${ }^{15}$ The appropriate level of glucose control and the best method to target glucose levels are still topics for research in the perioperative setting.

There are steps we can take to improve diabetes care in the perioperative setting. Patients who may benefit most may be those with diabetes who are scheduled for surgery requiring preoperative anesthetic assessment before the day of surgery and postoperative hospital admission. For this population, an elevated HbA1c can trigger further inhospital optimization of diabetes care. In some centres, including our own, an elevated $\mathrm{HbA} 1 \mathrm{c}$ results in the timely involvement of an endocrinologist to adjust diabetic medication therapy and to arrange further follow-up after hospital discharge. Although the intensity of intraoperative glycemic control is still uncertain, more frequent monitoring for and treatment of hyperglycemia in the operating room may facilitate postoperative glycemic control.

The use of HbA1c remains uncertain for patients with diabetes who are admitted for day surgery and for patients without a history of diabetes. For day surgery, the measurement is usually unavailable before hospital discharge so it is essential to forward this information to the patient's primary care provider (assuming the patient has one). For patients without a history of diabetes, the cost-benefit ratio of obtaining HbA1c levels for diagnostic screening is unclear. In such patients, if blood glucose is elevated, HbA1c may be useful if an abnormal result

Table Diagnostic characteristics for random and fasting blood glucose based on data from Koumpan et al. ${ }^{9}$

\begin{tabular}{|c|c|c|c|c|c|c|c|c|}
\hline Cutoff value & $\mathrm{TP}$ & FP & FN & $\mathrm{TN}$ & Sensitivity, \% (95\% CI) & Specificity, \% (95\% CI) & $\mathrm{LR}+(95 \% \mathrm{CI})$ & $\mathrm{LR}-(95 \% \mathrm{CI})$ \\
\hline \multicolumn{9}{|c|}{ Diagnosis of new diabetes in participants with no prior history $(\mathrm{HbA} 1 \mathrm{c} \geq 6.5 \%)$} \\
\hline $\mathrm{RBG} \geq 11.1 \mathrm{mmol} \cdot \mathrm{L}^{-1}$ & 2 & 1 & 11 & 314 & $15.4(2.7$ to 46.3$)$ & 99.7 (98.0 to 99.9$)$ & $48(4.7$ & 0.8 \\
\hline $\mathrm{FBG} \geq 7.0 \mathrm{mmol} \cdot \mathrm{L}^{-1}$ & 8 & 23 & 4 & 241 & 66.7 (35.4 to 88.7$)$ & $91.3(87.0$ to 94.3$)$ & $7.6(4.4$ to -13$)$ & $0.4(0.2$ to 0 . \\
\hline \multicolumn{9}{|c|}{ Diagnosis of suboptimal glycemic control in participants with diabetes $(\mathrm{HbA} 1 \mathrm{c}>7.0 \%)$} \\
\hline $\mathrm{RBG} \geq 11.1 \mathrm{mmol} \cdot \mathrm{L}^{-1}$ & 16 & 1 & 22 & 30 & 42.1 (26.7 to 59.0$)$ & 96.8 (81.5 to 99.8$)$ & 13 (1.8 to 93$)$ & $0.6(0.4$ to $\mathrm{C}$ \\
\hline $\mathrm{FBG} \geq 7.0 \mathrm{mmol} \cdot \mathrm{L}^{-1}$ & 31 & 16 & 5 & 14 & 86.1 (69.7 to 94.8$)$ & 46.7 (28.8 to 65.4$)$ & $1.6(1.1$ to 2.3$)$ & 0.3 (0.1 to 0.7 \\
\hline
\end{tabular}

Data for TP, FP, FN, and TN are $n / 332$ for participants with no prior history and $n / 70$ for participants with suboptimal control

$\mathrm{CI}=$ confidence interval; $\mathrm{FBG}=$ fasting blood glucose; $\mathrm{FN}=$ false negative; $\mathrm{FP}=$ false positive; $\mathrm{LR}+=$ positive likelihood ratio; $\mathrm{LR}-=$ negative likelihood ratio; $\mathrm{RBG}=$ random blood glucose; $\mathrm{TN}=$ true negative; $\mathrm{TP}=$ true positive 
triggers further workup and follow-up by an internist or endocrinologist. This is an area for research.

As perioperative physicians, we have a window of opportunity to advance the global management of diabetes in our preoperative clinics. Like smoking cessation, preoperative identification of either undiagnosed patients with diabetes or those with suboptimal control of diabetes provides an opportunity to refer patients to other resources to improve their long-term management. As perioperative physicians, we care about the effect of hyperglycemia on perioperative morbidity and mortality, but as perioperative physicians, we need to care about the effect of hyperglycemia on the long-term outcomes of this at-risk population. Hopefully, the results of the study by Koupam et al. will prompt us to be more vigilant in identifying patients with undiagnosed diabetes and patients with suboptimal glycemic control as part of our preoperative assessments. As perioperative physicians, we have a role to play in finding the sweet spot in diabetes management.

\section{Trouver le juste équilibre dans l'évaluation périopératoire}

Le diabète est une affection chronique qui s'accompagne d'une morbidité substantielle dans le monde entier. L'année dernière, la prévalence mondiale du diabète a été de 382 millions de personnes $(8,3 \%$ de la population mondiale); d'ici 2035, sa prévalence devrait atteindre 592 millions d'individus. ${ }^{1}$ Au Canada, un diabète a été diagnostiqué chez près de 2,4 millions de personnes, soit $6,7 \%$ de la population. Plus important encore, $20 \%$ des cas de diabète restent non diagnostiqués. ${ }^{2}$ En 2010, parmi les pays d'Amérique, d'Europe et d'Océanie, le Canada avait la troisième plus forte prévalence chez les adultes âgés de 20 à 79 ans. $^{2}$

Le coût du diabète pour le système de santé au niveau des individus et de la société est considérable. Le diabète et ses complications à long terme entraînent une augmentation des taux d'hospitalisation et de décès prématuré. ${ }^{2}$ L'Agence de la santé publique du Canada a estimé que le coût du diabète au niveau national avait été de 2,5 milliards de dollars en $2000^{2}$ Cette estimation prudente ne tenait pas compte des coûts engendrés par les complications à long terme du diabète ou des autres maladies chroniques qui lui étaient associées.

Dans un contexte périopératoire, le diabète est associé à une augmentation de la morbidité et de la mortalité, ${ }^{3-5}$ particulièrement en présence d'une hyperglycémie. L'identification en préopératoire des patients ayant un diabète non diagnostiqué et des patients diabétiques dont la glycémie est insuffisamment contrôlée peut guider les décisions concernant le contrôle glycémique périopératoire et à long terme. L'identification de ce dernier groupe est particulièrement importante dans la mesure où des études antérieures ont montré une baisse du risque de complications microvasculaires lorsque le contrôle glycémique est amélioré sur le long terme. ${ }^{6}$ Malheureusement, l'obtention d'un contrôle glycémique satisfaisant reste difficile. Dans une étude canadienne, seulement $39 \%$ des participants avaient maintenu le taux cible d'hémoglobine glycosylée A1c (HbA1c) au taux cible de $7 \%$ ou à un taux inférieur sur une période d'un an. ${ }^{7}$ Dans une étude de cohorte, seulement $22,4 \%$ des patients ayant un diabète mal contrôlé qui avaient été hospitalisés ont bénéficié d'une modification quelconque de leur prise en charge diabétique avant de recevoir leur congé. ${ }^{8}$

Dans ce numéro, Koumpan et coll. présentent une étude observationnelle prospective dont le but était de déterminer la prévalence de l'hyperglycémie chronique chez des patients préopératoires sans diagnostic de diabète, la bonne qualité du contrôle glycémique chez des patients diabétiques connus en préopératoire et les caractéristiques utiles de diagnostic des glycémies aléatoires et des glycémies à jeun pour l'identification des patients ayant un mauvais contrôle glycémique. ${ }^{9}$ Les participants étaient des adultes devant subir une évaluation à la clinique préopératoire du site d'étude en prévision d'une chirurgie élective. Une HbA1c d'au moins 6,5\% a été utilisée pour poser un diagnostic temporaire de diabète et servir de référence aux autres dosages du contrôle glycémique. Le diagnostic de diabète était basé sur les valeurs de HbA1c recommandées par l'Association canadienne du diabète. ${ }^{10}$

La cohorte était constituée de 332 (82,6\%) participants sans diagnostic de diabète et de $70(17,4 \%)$ participants ayant un diabète connu. Parmi les participants sans diagnostic de diabète, les investigateurs ont trouvé que « 23,2 \% (77/332) d'entre eux avaient un risque très élevé de développer un diabète (prédiabétiques) et que 3,9\% (13/332) avaient un diagnostic probable de diabète » sur la base des valeurs d'HbA1c. ${ }^{9}$ Parmi les participants ayant un diabète connu, 55,7 \% (39/70) avaient un contrôle glycémique sous-optimal, ayant un taux d'HbA1c supérieur à $7 \%$. Ces résultats confirment la prévalence du prédiabète et du diabète non diagnostiqués décrits dans des études périopératoires antérieures. ${ }^{11,12}$ La prévalence d'un contrôle glycémique sous-optimal chez les patients diabétiques est comparable à celle observée dans des études épidémiologiques précédentes (49\% au Canada, $63,0 \%$ aux États-Unis) ${ }^{13,14}$ et souligne le défi permanent que constitue le contrôle glycémique optimal à long terme.

Le Tableau montre les caractéristiques diagnostiques de la glycémie aléatoire et de la glycémie à jeun au cours de 
Tableau Caractéristiques diagnostiques pour la glycémie aléatoire et la glycémie à jeun d'après les données de Koumpan et coll. ${ }^{9}$

Valeur seuil

VP $\quad$ FP $\quad$ FN VN $\quad$ Sensibilité, \% (IC à $95 \%) \quad$ Spécificité, \% (IC à $95 \%) \quad$ LR+ (IC à $95 \%) \quad$ LR- (IC à $95 \%)$

Nouveau diagnostic de diabète chez des participants sans antécédents (HbAlc $\geq 6,5 \%$ )

$\mathrm{Gal} \geq 11,1 \mathrm{mmol} \cdot \mathrm{L}^{-1} \quad 2 \quad 1 \quad 11 \quad 314 \quad 15,4(2,7$ à 46,3$)$

$99,7(98,0$ à 99,9$)$

48 (4,7 à 501)

$0,8(0,7$ à 1,1$)$

GàJ $\geq 7,0 \mathrm{mmol} \cdot \mathrm{L}^{-1} \quad 8 \quad 23 \quad 4 \quad 241 \quad 66,7(35,4$ à 88,7$)$

$91,3(87,0$ à 94,3$)$

7,6 (4,4 à 13)

$0,4(0,2$ à 0,8$)$

Diagnostic de contrôle glycémique sous-optimal chez les participants diabétiques (HbA1c $>7,0 \%$ )

$\begin{array}{llrrrrrrr}\mathrm{Gal} \geq 11,1 \mathrm{mmol} \cdot \mathrm{L}^{-1} & 16 & 1 & 22 & 30 & 42,1(26,7 \text { à } 59,0) & 96,8(81,5 \text { à } 99,8) & 13(1,8 \text { à } 93) & 0,6(0,4 \text { à } 0,8) \\ \mathrm{GàJ} \geq 7,0 \mathrm{mmol} \cdot \mathrm{L}^{-1} & 31 & 16 & 5 & 14 & 86,1(69,7 \text { à } 94,8) & 46,7(28,8 \text { à } 65,4) & 1,6(1,1 \text { à } 2,3) & 0,3(0,1 \text { à } 0,7)\end{array}$

Les données concernant les VP, FP, FN et VN sont $n / 332$ pour les participants sans antécédents et $n / 70$ pour les participants avec un contrôle sous-optimal

$\mathrm{IC}=$ intervalle de confiance; GàJ $=$ glycémie à jeun; FN $=$ faux négatif; $\mathrm{FP}=$ faux positif; $\mathrm{LR}+=$ ratio de probabilité positif; $\mathrm{LR}-=$ ratio de probabilité négatif; $\mathrm{Gal}=$ glycémie aléatoire; $\mathrm{VN}=$ vrai négatif; $\mathrm{VP}=$ vrai positif

cette étude. Quand les patients sans diagnostic antérieur de diabète ont été évalués selon les critères d'HbA1c, un nouveau diagnostic de diabète pouvait être déduit en présence de tests positifs, c'est-à-dire, lorsque la glycémie aléatoire et la glycémie à jeun étaient toutes deux spécifiques du diabète. Aucun des deux tests n'était sensible: le diagnostic de diabète ne peut pas être éliminé en présence d'un test négatif. Quand les patients ayant un antécédent de diabète ont été évalués selon les critères d'HbA1c, la glycémie aléatoire était spécifique mais non sensible pour le contrôle glycémique chronique sous-optimale, alors que la glycémie à jeun était sensible mais non spécifique. Les chiffres sont petits dans la plupart des catégories diagnostiques (vrai positif, faux positif, faux négatif et vrai négatif) et entraînent de grands intervalles de confiance pour l'estimation des caractéristiques diagnostiques; aussi, l'interprétation de ces résultats doit être prudente.

Plusieurs conclusions peuvent être tirées si les constatations de cette étude sont confirmées par de plus grandes cohortes. Tout d'abord, chez les patients sans antécédents de diabète, une élévation de la glycémie aléatoire ou de la glycémie à jeun est fortement suspecte de diabète et devrait mener à une évaluation diagnostique ainsi qu'un suivi complémentaires. Toutefois, l'absence de résultats augmentés ne doit pas rassurer, en particulier chez des patients ayant des facteurs de risque de diabète ${ }^{9}$ Deuxièmement, parmi les patients ayant un diabète connu, aucun des deux tests seul n'est suffisant pour déterminer le niveau de contrôle glycémique. Cette observation est en accord avec la gestion du diabète en dehors d'un cadre péri-opératoire ou un seul dosage de la glycémie est insuffisant pour déterminer le statut du contrôle glycémique chronique. L'hémoglobine glycosylée reste un meilleur test pour le diagnostic du diabète et pour la détermination de la qualité du contrôle glycémique à long terme.

En tant qu'anesthésiologistes, nous pourrions être tentés de minimiser les implications de cette étude. Même avec
402 participants, la taille de l'échantillon est petite. Les directives canadiennes recommandent la glycémie à jeun qui est un examen rapide et bon marché, comparativement à l'HbA1c. ${ }^{15}$ Le niveau acceptable de contrôle glycémique et la meilleure méthode permettant de contrôler les taux de glucose reste des sujets de recherche dans le cadre périopératoire.

Il existe des mesures que nous pouvons adopter pour améliorer les soins diabétiques de ce contexte périopératoire. Les patients susceptibles d'en bénéficier le plus sont les patients diabétiques pour lesquelles une intervention chirurgicale programmée nécessite une évaluation anesthésique préopératoire, le jour avant l'intervention et un séjour hospitalier en postopératoire. Pour cette population, un pourcentage élevé d'HbA1c peut déclencher une optimisation des soins diabétiques en milieu hospitalier. Dans certains centres, dont le nôtre, une élévation de l'HbA1c entraîne l'implication en temps opportun d'un endocrinologue qui adaptera le traitement médicamenteux antidiabétique et veillera à organiser un suivi complémentaire après le congé de l'hôpital. Bien que l'intensité du contrôle glycémique peropératoire reste incertaine, une surveillance plus fréquente à la recherche de l'hyperglycémie et son traitement au bloc opératoire peuvent faciliter le contrôle glycémique postopératoire.

L'utilisation de l'HbA1c reste incertaine pour les patients diabétiques hospitalisés pour une chirurgie d'un jour et pour les patients sans antécédents de diabète. Pour la chirurgie d'un jour, le dosage est habituellement pas disponible avant le congé de l'hôpital; il est donc essentiel de faire suivre cette information au médecin de famille du patient (en supposant que le patient en ait un). Pour les patients sans antécédents de diabète, le rapport bénéfice - coût du dosage de l'HbA1c pour le dépistage diagnostique n'est pas clairement établi. Si la glycémie est élevée chez ces patients, l'HbA1c peut-être utile si un résultat anormal déclenche un bilan plus approfondi et un suivi par un médecin interniste ou un endocrinologue. Il s'agit là d'un domaine de recherche. 
En tant que médecins de la phase périopératoire, nous disposons d'une période très propice pour faire avancer la prise en charge globale du diabète dans nos cliniques préopératoires. Comme l'arrêt du tabagisme, l'identification préopératoire de patients dont le diabète n'a pas été diagnostiqué ou dont le contrôle diabétique est sous optimal, nous donne une chance de référer ces patients à d'autres ressources afin d'améliorer leur prise en charge à long terme. En tant que médecins de la phase périopératoire, nous nous préoccupons de l'effet de l'hyperglycémie sur la morbidité et la mortalité périopératoire, mais en tant que médecins de la phase périopératoire, nous devons nous préoccuper de l'effet de l'hyperglycémie sur l'évolution à long terme de cette population à risque. Nous pouvons espérer que les résultats de l'étude de Koupam et coll. nous inciteront à être plus vigilants dans l'identification des patients ayant un diabète non diagnostiqué et des patients ayant un contrôle glycémique sous optimal, en les incluant dans nos évaluations préopératoires. En tant que médecins de la phase périopératoire, nous avons un rôle à jouer pour trouver le bon équilibre dans la gestion du diabète.

Conflicts of interest None declared.

Conflits d'intérêts Aucun déclaré.

\section{References}

1. International Diabetes Federation. IDF Diabetes Atlas, Sixth edition - 2013. Available from URL: www.idf.org/diabetesatlas (accessed February 2014).

2. Public Health Agency of Canada. Diabetes in Canada: Facts and Figures From a Public Health Perspective - 2011. Available from URL: http://www.phac-aspc.gc.ca/cd-mc/publications/diabetesdiabete/facts-figures-faits-chiffres-2011/ (accessed February 2014).
3. Barone BB, Yeh HC, Snyder CF, et al. Postoperative mortality in cancer patients with preexisting diabetes: systematic review and meta-analysis. Diabetes Care 2010; 33: 931-9.

4. Zhang $X, W u Z$, Peng $X$, et al. Prognosis of diabetic patients undergoing coronary artery bypass surgery compared with nondiabetics: a systematic review and meta-analysis. J Cardiothorac Vasc Anesth 2011; 25: 288-98.

5. Tsang ST, Gaston P. Adverse peri-operative outcomes following elective total hip replacement in diabetes mellitus: a systematic review and meta-analysis of cohort studies. Bone Joint J 2013; 95-B: 1474-9.

6. Canadian Diabetes Association Clinical Practice Guidelines Expert Committee. Targets for glycemic control. Can J Diabetes 2013; 37: S31-4.

7. Braga MF, Casanova A, Teoh $\mathrm{H}$, et al. Poor achievement of guidelines-recommended targets in type 2 diabetes: findings from a contemporary prospective cohort study. Int J Clin Pract 2012; 66: 457-64

8. Griffith ML, Boord JB, Eden SK, Matheny ME. Clinical inertia of discharge planning among patients with poorly controlled diabetes mellitus. J Clin Endocrinol Metab 2012; 97: 2019-26.

9. Koumpan $Y$, VanDenKerkhof E, van Vlymen J. An observational cohort study to assess glycosylated hemoglobin screening for elective surgical patients. Can J Anesth 2014; 61: this issue. DOI:10.1007/s12630-014-0124-y.

10. Canadian Diabetes Association Clinical Practice Guidelines Expert Committee. Definition, classification and diagnosis of diabetes, prediabetes and metabolic syndrome. Can J Diabetes 2013; 37: S8-11.

11. McGinn JT Jr, Shariff MA, Bhat TM, et al. Prevalence of dysglycemia among coronary artery bypass surgery patients with no previous diabetic history. J Cardiothorac Surg 2011; 6: 104.

12. Hatzakorzian R, Bui H, Carvalho G, Shan WL, Sidhu S, Schricker $T$. Fasting blood glucose levels in patients presenting for elective surgery. Nutrition 2011; 27: 298-301.

13. Saydah AH, Fradkin J, Cowie CC. Poor control of risk factors for vascular disease among adults with previously diagnosed diabetes. JAMA 2004; 291: 335-42.

14. Harris SB, Ekoe JM, Zdanowicz Y, Webster-Bogaert S. Glycemic control and morbidity in the Canadian primary care setting (results of the diabetes in Canada evaluation study). Diabetes Res Clin Pract 2005; 70: 90-7.

15. Merchant $R$, Chartrand D, Dain $S$, et al. Guidelines to the practice of anesthesia revised edition 2013. Can J Anesth 2013; 60: 60-84. 